\title{
H $\alpha$ Doppler shifts in a tornado in the solar corona
}

\author{
B. Schmieder ${ }^{1}$, P. Mein ${ }^{1}$, N. Mein ${ }^{2}$, P. J. Levens ${ }^{3}$, N. Labrosse ${ }^{3}$, and L. Ofman ${ }^{4}$ \\ ${ }^{1}$ LESIA, Observatoire de Paris, PSL Research University, CNRS, Sorbonne Universités, UPMC Univ. Paris 06, Univ. Paris Diderot, \\ Sorbonne Paris Cité, 5 place Jules Janssen, 92195 Meudon, France \\ e-mail: brigitte.schmieder@obspm.fr \\ 2 Observatoire de Paris, 61 avenue de l'Observatoire, 75014 Paris, France \\ 3 SUPA School of Physics and Astronomy, University of Glasgow, Glasgow, G12 8QQ, UK \\ ${ }^{4}$ CUA and NASA Goddard Space Flight Center, Code 671, Greenbelt, MD 20771, USA
}

Received 22 April 2016/ Accepted 28 November 2016

\begin{abstract}
Context. High resolution movies in $193 \AA$ from the Atmospheric Imaging Assembly (AIA) on the Solar Dynamic Observatory (SDO) show apparent rotation in the leg of a prominence observed during a coordinated campaign. Such structures are commonly referred to as tornadoes. Time-distance intensity diagrams of the AIA data show the existence of oscillations suggesting that the structure is rotating.

Aims. The aim of this paper is to understand if the cool plasma at chromospheric temperatures inside the tornado is rotating around its central axis.

Methods. The tornado was also observed in $\mathrm{H} \alpha$ with a cadence of $30 \mathrm{~s}$ by the MSDP spectrograph, operating at the Solar Tower in Meudon. The MSDP provides sequences of simultaneous spectra in a 2D field of view from which a cube of Doppler velocity maps is retrieved.

Results. The H $\alpha$ Doppler maps show a pattern with alternatively blueshifted and redshifted areas of 5 to $10^{\prime \prime}$ wide. Over time the blueshifted areas become redshifted and vice versa, with a quasi-periodicity of 40 to $60 \mathrm{~min}$. Weaker amplitude oscillations with periods of 4 to 6 min are superimposed onto these large period oscillations.

Conclusions. The Doppler pattern observed in $\mathrm{H} \alpha$ cannot be interpreted as rotation of the cool plasma inside the tornado. The $\mathrm{H} \alpha$ velocity observations give strong constraints on the possible interpretations of the AIA tornado.
\end{abstract}

Key words. Sun: filaments, prominences

\section{Introduction}

The term solar tornadoes has been used to describe apparently rotating magnetic structures above the solar limb, as seen in high resolution images and movies from the Atmospheric Imaging Assembly (AIA) aboard the Solar Dynamics Observatory (SDO; Lemen et al. 2012). Movies obtained with high spatial and temporal resolution have enabled the discovery of the incredible dynamic nature of prominences. Even in quiescent prominences, apparently rotating, tornado-like structures, apparent upflows and downflows in quasi-vertical structures, and rising bubbles are seen (Dudík et al. 2012; Orozco Suárez et al. 2012; Wedemeyer et al. 2013; Berger 2014; Su et al. 2014). Spectroscopy is necessary to analyse the real plasma motion and physical conditions of the tornado. An example of tornado rotation around its axis was detected in hot plasma $\left(T>10^{6} \mathrm{~K}\right)$ surrounding the prominence legs by the Extreme-ultraviolet Imaging Spectrometer (EIS) on Hinode (Kosugi et al. 2007) using Doppler shifts in a number of coronal lines (Su et al. 2014; Levens et al. 2015). We ask here whether or not the cool material $\left(T \sim 10^{4} \mathrm{~K}\right)$ inside the tornado, which is visible in chromospheric lines, also presents signatures of rotation and, if so, with what velocity?

Wedemeyer et al. (2013) showed some examples of tornadolike structures observed with the Swedish Solar Telescope (SST) in the $\mathrm{H} \alpha$ line and posed the question: Are they the legs of prominences? Their first example is an observation of a filament on the disc. Blueshifts and redshifts are observed along the axis of the structure that could give the impression of a rolling flux rope. Their other example is a prominence observed above the limb. Torsional motions, such as those observed during an eruption, are detected along fine threads. These two examples were not conclusive concerning these apparent tornado-like structures. New ground-based observations were required to investigate these prominence motions further.

Mghebrishvili et al. (2015) explored the oscillations seen in tornadoes observed with coronal AIA filters (171 $\AA, 211 \AA$, $193 \AA)$. They concluded that they could see two kinds of patterns depending on the time. During the first phase the tornadoes were rising with a twisting pattern, while later the tornadoes stopped their rise and their oscillations were interpreted as due to magneto-hydro-dynamic (MHD) kink waves with one end fixed in the photosphere and an open end at the top, or by the rotation of two tornado structures during a quasi-periodic phase.

Panasenco et al. (2014) explained the apparent vortical motion in prominence spines and barbs exhibited in TRACE and SDO/AIA $171 \AA$ images and movies as counterstreaming flows giving the illusion of rotation. The apparent rotational motion is only observed in $2 \mathrm{D}$ projection at the limb in the plane of the sky. The authors claimed, "the constant counterstreaming motion of the prominence plasma along the thin threads, especially when they connect the vertical parts of the prominence (legs and barbs) to the much more horizontal spine, creates an effect that the eye associates with rotation". They concluded that the tornado-like structure oscillates, but does not rotate. 
Oscillations in prominences are frequently observed and are important for prominence seismology because of the possible role of MHD waves in heating the prominence material (Ofman et al. 1998, 2015). With the high temporal and spatial resolution of recent solar telescopes, it has been possible to resolve small-scale oscillations and waves (Engvold 2008). Transverse oscillations have been reported recently using Hinode/SOT and SDO/AIA (see papers of Okamoto et al. 2007, 2015; Schmieder et al. 2013). These oscillations, which are commonly observed as density fluctuations, concern either horizontal fine threads, with periods of the order of 10 to $15 \mathrm{~min}$ (Okamoto et al. 2015), or feet of prominences, with periods of 5 min (Schmieder et al. 2014b). Ofman et al. (2015) interpret the transverse oscillations observed in a feet of prominence as due to non-linear fast magnetosonic waves. They also explore non-linear gravitational MHD oscillations of heavy material in prominence feet, supported by a dipped magnetic field structure using a 2.5D MHD model and find lesser agreement with these global waves owing to their longer periods. The transverse oscillations in fine horizontal threads have been interpreted as being Alfvén waves, coupled with kink waves (Antolin et al. 2015). However, these oscillations and waves have smaller periods as the typical tornado period of 1.5 to $2 \mathrm{~h}$.

An interesting spectroscopic approach to the problem of rotation was taken by Orozco Suárez et al. (2012) looking at a tornado for one hour with the Tenerife Infrared Polarimeter (TIP) instrument operating at the Vacuum Tower Telescope (VTT) in the Canary Islands. They used the He I infrared multiplet. Surprisingly the two components at $1082.909 \mathrm{~nm}$ and $1083.029 \mathrm{~nm}$ have an opposite behaviour in terms of Doppler shifts. Both components have a low intensity signal, a weak line width, and are optically thin. These authors concluded that there are possible rotational signatures at the edges of the tornado because in the Doppler shift versus time diagrams the cells of blueshifts are consistently located at the right edge of the leg, and the redshifts at the left edge of the leg. However this interpretation needs to be supported by more observations with similar characteristic Doppler shifts.

The apparent upflows and downflows in prominence legs do not prove that the structure is vertical. Using $\mathrm{H} \alpha$ spectra obtained with the Multi-channel Subtractive Double Pass (MSDP) instrument in the Meudon Solar Tower it was shown that in prominences the velocity vectors were not aligned with the apparent vertical intensity structures, as the movies suggest, but have a significant angle with respect to the vertical. This suggests the existence of a more or less horizontal magnetic support rather than vertical flows (Schmieder et al. 2010). In an analysis of a hedgerow prominence, Chae (2010) suggested a similar magnetic support. The descending observed knots are basically supported against gravity by horizontal magnetic fields even when they descend, and the complex variations of their descending speeds should be attributed to small imbalances between gravity and force of magnetic tension.

The French telescope Télescope Héliographique pour l'Étude du Magnétisme et des Instabilités Solaires (THEMIS) in the Canary Islands with its MulTi-Raies (MTR) mode has been observing prominences during international campaigns since 2012. More than 200 prominences have been observed in the $\mathrm{He} \mathrm{I}_{3}$ line. Statistical work has been presented (López Ariste 2015) and case studies have been published (Schmieder et al. $2013,2014 b$ ). The main result is that the magnetic field is mainly horizontal in prominences. Recently the magnetic field of tornado-like structures observed by THEMIS has been analysed in 2D maps (Schmieder et al. 2015; Levens et al. 2016a,b).
Their histogram shows a primary horizontal component with two secondary peaks that are not easy to interpret. The field strength could reach 40 gauss in some parts of a tornado. From the theoretical point of view we can quote the MHD model of Luna et al. (2015), which was based on the modelling of a vertical cylinder with a vertical field along its central axis and a more and more helical field as closer to the periphery. It is difficult to model such a structure and obtain the corresponding Stokes parameters that have been observed.

However, as has been mentioned, tornadoes are often considered to be legs of prominences (Wedemeyer et al. 2013; Levens et al. 2016a). Many static models proposed that legs or barbs of prominences are piles of dips in magnetic field lines supporting the cool plasma (Aulanier \& Demoulin 1998; Dudík et al. 2008; Heinzel \& Anzer 1999; Mackay et al. 2010). More recently simulations of filament formations by condensation have supported this idea (Xia et al. 2014; Terradas et al. 2015).

In this paper we present a tornado-like prominence observed by AIA in $193 \AA$ on September 24, 2013, where it looks like it is rotating as a vertical structure with a characteristic period of around 90 min (Sect. 2 and movie), and in $\mathrm{H} \alpha$ with observations made by the MSDP spectrograph (Sect. 3). Our aim is to compute the velocities along the line of sight of the cool plasma to see if we observe similar rotational signatures to those seen with AIA. In the last section, we discuss possible interpretations of the observed oscillatory behaviour of the $\mathrm{H} \alpha$ Doppler shifts for the AIA tornado.

\section{AIA tornado}

In the $193 \AA$ spectral window of AIA the tornado, or leg of the prominence, is seen as a silhouette in absorption on September 24, 2013. This absorption is mainly from neutral hydrogen and neutral/ionized helium seen against a bright background (Schmieder et al. 2004; Anzer \& Heinzel 2005) (Figs. 1, 2). The tornado height is about $45 \mathrm{Mm}$. Its width varies with time and height above the photosphere. Sometimes it splits into two or more threads and shows lateral extension, as seen in Fig. 2. Generally this tornado is narrower near the footpoint and wider at the top, as is noted by Mghebrishvili et al. (2015). In order to study the temporal dynamics of the tornado, we construct time-distance diagrams at two different heights above the limb. The location of these cuts is shown with green lines in Fig. 2. The first cut is located at a height of $20 \mathrm{Mm}$ and the second cut is around $30 \mathrm{Mm}$. The bottom panels of Fig. 2 show the timedistance diagrams. There are apparent quasi-periodic transverse displacements of the axis during the interval of time $(8 \mathrm{~h})$. The period and amplitude of the displacement is difficult to compute because many different structures are visible. The period starts off around $80 \mathrm{~min}$, and then increases to about $110 \mathrm{~min}$ at later times (from the lower cut). A mean period of $90 \mathrm{~min}$ is a reasonable value. The tornado looks as if it is a double structure at the top, which is similar to that seen in Mghebrishvili et al. (2015). According to the amplitude of the oscillations, we estimate a velocity of $7 \mathrm{~km} \mathrm{~s}^{-1}$.

\section{3. $\mathrm{H} \alpha$ data}

The tornado is part of a prominence that was observed as a filament for a few days in survey images from Meudon. The filament is oriented along a north-south meridian. The filament was the target of a coordinated campaign as it was crossing the 
B. Schmieder et al.: $\mathrm{H} \alpha$ Doppler shifts in a tornado in the solar corona
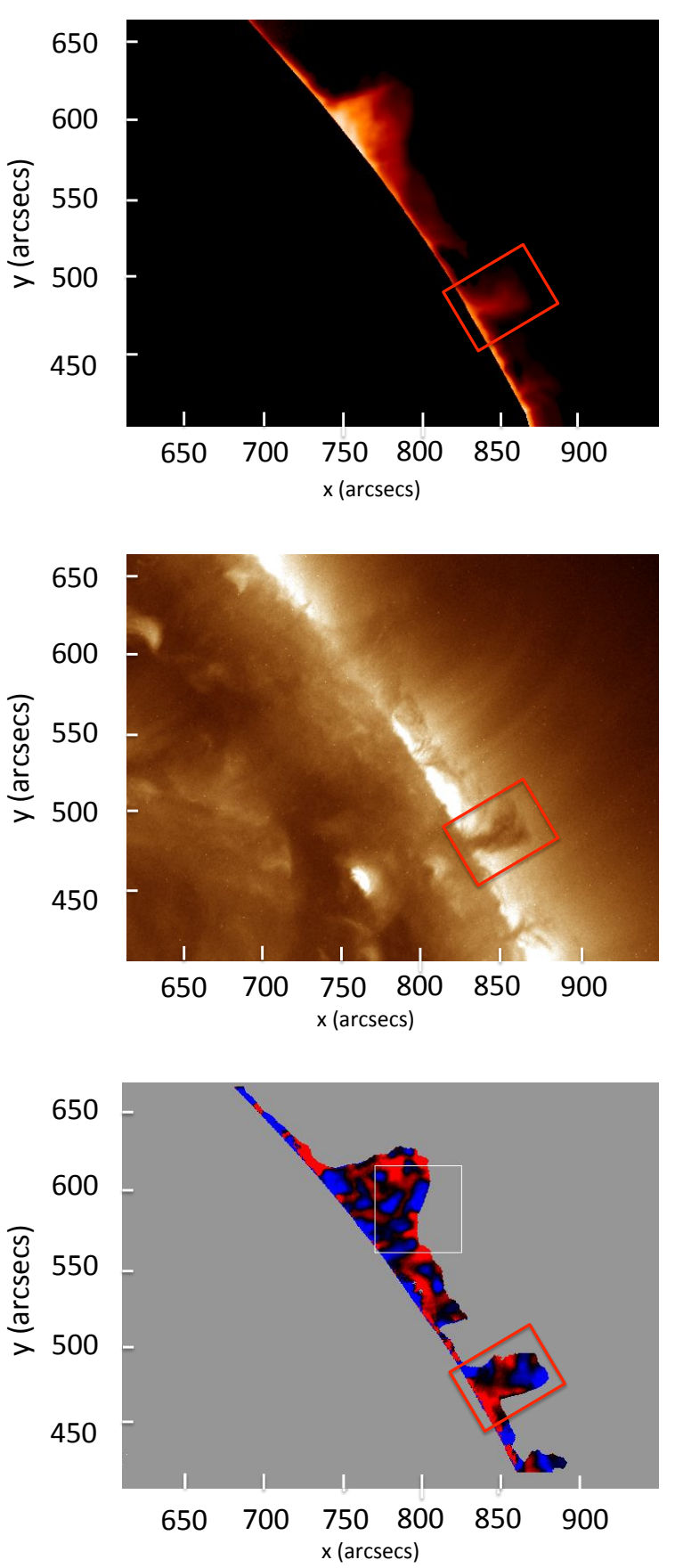

Fig. 1. Prominence observed on September 24, 2013 at 12:22 UT. Top panel: $\mathrm{H} \alpha$ image from the MSDP instrument operating on the Solar Tower in Meudon. Middle panel: SDO/AIA $193 \AA$ image. The prominence in $193 \AA$ is observed in absorption. Bottom panel: $\mathrm{H} \alpha$ Doppler shift map. The tornado is in the red box, which corresponds to the field of view of the top panels of Figs. 4-7. The white box indicates the part of the prominence observed by IRIS (Schmieder et al. 2014b).

limb on September 24, 2013, with coordination between groundbased instruments and the spectrograph aboard the space mission IRIS.

Time sequences of $\mathrm{H} \alpha$ MSDP observations obtained at the Meudon Solar Tower have already been analysed in Schmieder et al. (2014a) and Heinzel et al. (2015). These two papers are focused on the part of the prominence observed by IRIS (Fig. 1, white box). Here we consider the tornado-like
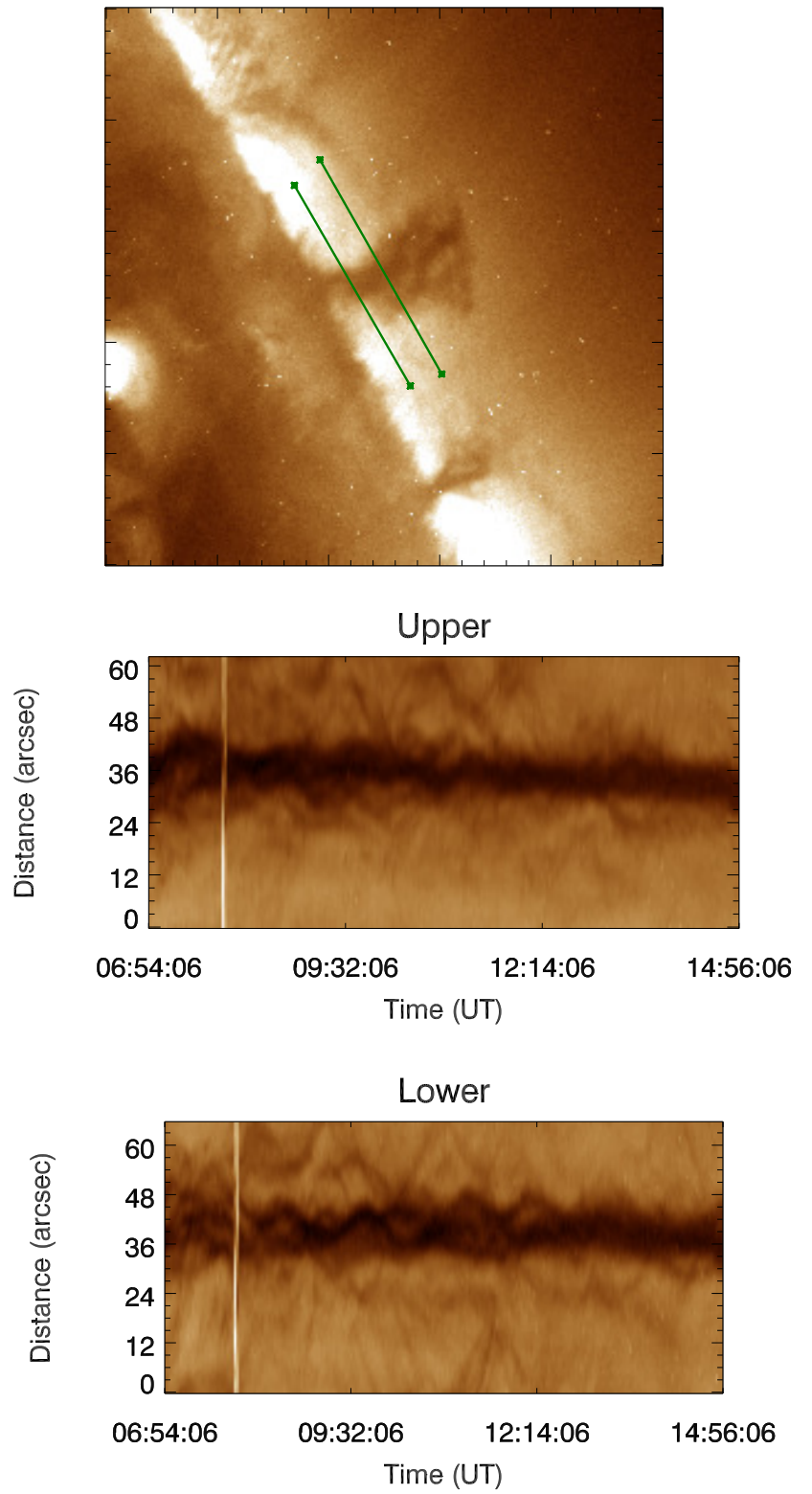

Fig. 2. Prominence observed on September 24, 2013 by SDO/AIA in the $193 \AA$ window. Top panel: image from AIA at 06:54 UT. Field of view is approximately $200^{\prime \prime} \times 200^{\prime \prime}$. The green lines show where the cuts in the bottom panels were measured. Bottom panels: time-distance intensity diagrams for the two cuts between 06:54 UT and 14:56 UT $(8 \mathrm{~h})$. The distance between the two cuts is approximately $10 \mathrm{Mm}$.

structure located in the lower part of the field of view (Fig. 1, red box).

\subsection{MSDP data processing}

The MSDP is a spectrograph with a wide slit acting as a field stop (Mein 1991). In the Meudon MSDP, the size of the elementary field of view is $465^{\prime \prime} \times 60^{\prime \prime}$. The pixel size is $0.6^{\prime \prime}$. An optical system using prisms before the entrance window scans the target by five successive images with small overlaps for adjustments by cross-correlations. The result is a $450^{\prime \prime} \times 260^{\prime \prime}$ full map recorded within $30 \mathrm{~s}$. The size of the field can be slightly different according to the spatial shifts deduced from correlations between elementary fields and depending on seeing and coelostat motions. The observations are obtained in an $(x, y)$ non-heliographic 

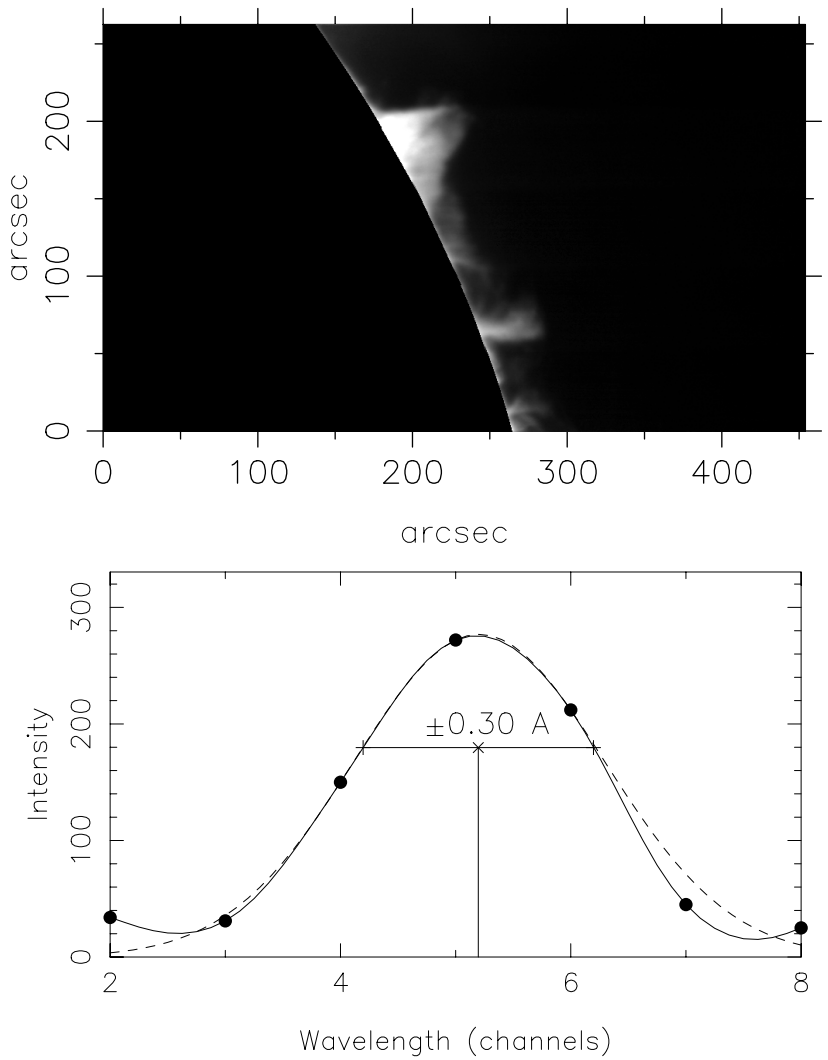

Fig. 3. Top panel: full field-of-view image $\left(450^{\prime \prime} \times 260^{\prime \prime}\right)$ of the MSDP in non-heliographic coordinates $(x, y)$. Bottom panel: example of interpolation of MSDP $\mathrm{H} \alpha$ profiles by the cubic method (solid line) and by a Gaussian function (dashed line) and determination of Doppler shifts via a bisector method.

coordinate system. The top panel of Fig. 3 shows the map of prominence intensities at 12:22 UT.

In each of the nine channels of elementary frames the wavelength is approximately constant along $x$, but varies along $y$. The wavelength shift between two successive channels is $\Delta \lambda=$ $0.30 \AA$ A In channels 1 and 9, located practically outside the emission line, the $\mathrm{H} \alpha$ prominence is not visible anymore. The data processing includes geometrical calibrations to locate the same solar points in all channels, and photometric calibrations providing continuity between overlaps of successive channels. Figure 3 bottom panel shows a typical prominence profile from channels 2 to 8 . It is defined by a cubic interpolation. Doppler shifts are determined by a bisector method between points of equal intensity and wavelength distance,

$D_{k}=k \Delta \lambda$.

In this paper, observations are processed with $k=2$. Prominence pixels are selected by bisector intensities larger than a given threshold (10\% of line centre disc intensity near the limb).

\subsection{Typical velocity amplitudes}

The zero Doppler shift is determined by using a mean value across the full prominence visible in the $450^{\prime \prime} \times 260$ " field of view, including the upper part of the prominence, north of the tornado (Fig. 3, top panel). In this way, we obtain accurate velocities relative to a structure that is much more extended than the tornado itself.

To estimate the relative accuracy of obtained Doppler velocities $v$, we need an estimate of typical amplitudes. We take as an
Table 1. Root mean squares of departures between measured velocities in $\mathrm{km} \mathrm{s}^{-1}$ obtained with cubic and Gaussian interpolations for strips of different distances from the solar limb.

\begin{tabular}{lcccc}
\hline \hline & $15^{\prime \prime}$ & $25^{\prime \prime}$ & $35^{\prime \prime}$ & $45^{\prime \prime}$ \\
\hline $\operatorname{rms}\left[V(x, y, t)-V_{\text {Gauss }}(x, y, t)\right]$ & 0.48 & 0.37 & 0.23 & 0.27 \\
\hline
\end{tabular}

example the time sequence starting at 12:06 UT and ending at 12:21 UT. For each time, we can compute the root mean square of measured velocities over the full field of the prominence as follows:

$\sigma(t)=\left(\left\langle V(x, y, t)^{2}\right\rangle_{x, y}\right)^{1 / 2}$.

The $\sigma(t)$ values are averaged over the 30 maps of the time sequence to provide the typical amplitude

$A=\langle\sigma(t)\rangle_{t}=2.16 \mathrm{~km} \mathrm{~s}^{-1}$.

The accuracy of the result also depends on the zero level of the Doppler shifts. We consider the differences between the average velocities of successive maps and their average over the same sequence. The corresponding rms is

$\delta v_{0}=0.11 \mathrm{~km} \mathrm{~s}^{-1}$.

It is less than $5 \%$ of the typical velocity amplitude and can be neglected as a first approximation.

\subsection{Comparison with Gaussian interpolation}

It is interesting to check whether velocity measurements are dependent on the interpolation method. Figure 3 shows the bisector method applied to a tornado line profile with cubic spline interpolation (solid line). We added a dashed line showing the Gaussian function determined by the three points of the highest intensities (channels 4-6). In this example, the agreement is very good in the core of the line near the points where the bisector method determines the Doppler shift.

To get more quantitative values, we compared departures for all points of the time sequence starting at 12:06 UT. Table 1 gives the root mean squares of departures in four strips parallel to the solar limb. Each strip is $10^{\prime \prime}$ wide. The distances from solar limb to strip centres are increasing from $15^{\prime \prime}$ to $45^{\prime \prime}$.

We see that the departures are globally decreasing with distance from the limb, until values less than $0.3 \mathrm{~km} \mathrm{~s}^{-1}$, that is only $14 \%$ of typical velocity amplitudes.

Slightly higher values are observed close to the limb. They may be due to profiles, including several solar structures, or velocity gradients that cannot be represented by only one Gaussian profile.

\subsection{Upper estimate of data noise effects}

It is possible to get upper estimates of data noise effects by comparing measured velocities in neighbouring points in time and space. We consider four points of the tornado (see Fig. 7 Sect. 3.7) during the full time sequence from 12:06 UT to 12:53 UT.

Table 2 shows the root mean squares of departures between measured velocities in $\mathrm{km} \mathrm{s}^{-1}$ at successive times $t$ and $t+\mathrm{d} t$, and neighbouring points $x$ and $x+\mathrm{d} x$ or $y$ and $y+\mathrm{d} y$, where $\mathrm{d} t$ is almost always equal to $30 \mathrm{~s}$ and $\mathrm{d} x=\mathrm{d} y$ equal to $0.5^{\prime \prime}$.

Departures corresponding to displacements $\mathrm{d} x$ and $\mathrm{d} y$ are low, and less than $0.3 \mathrm{~km} \mathrm{~s}^{-1}$ or $14 \%$ of typical amplitudes. Departures corresponding to successive times are larger. We see 
Table 2. Root mean squares of departures between measured velocities in $\mathrm{km} \mathrm{s}^{-1}$ at neighbouring times and positions for 4 tornado points A-D (see Fig. 7).

\begin{tabular}{lcccc}
\hline \hline & $\mathrm{A}$ & $\mathrm{B}$ & $\mathrm{C}$ & $\mathrm{D}$ \\
\hline $\operatorname{rms}[V(x, y, t+\mathrm{d} t)-V(x, y, t)]$ & 0.53 & 0.66 & 0.53 & 0.65 \\
$\operatorname{rms}[V(x+\mathrm{d} x, y, t)-V(x, y, t)]$ & 0.15 & 0.27 & 0.17 & 0.27 \\
$\operatorname{rms}[V(x, y+\mathrm{d} y, t)-V(x, y, t)]$ & 0.13 & 0.27 & 0.18 & 0.26 \\
\hline
\end{tabular}

later (Fig. 7, Sect. 3.7) that velocities versus time are not really stochastic. Evolving solar structures can account for such departures.

It is also possible to estimate noise effects directly with a CCD camera, although it depends very much on the profile intensity from pixel to pixel. In the case of Fig. 3, the corresponding signal-to-noise ratio at the intensity level of points used in the bisector method is around 60. Since the slope of line profile is near $0.17 \mathrm{~km} \mathrm{~s}^{-1}$ for $1 \%$ relative intensity fluctuations, and since the Dopplershift is deduced from the half sum of 2 wavelengths, we can estimate CCD noise effects at $0.20 \mathrm{~km} \mathrm{~s}^{-1}$. This value is slightly reduced, in fact, if we take interpolations used in data reduction into account.

Finally, we can conclude that effects due to data noise are probably smaller than $0.3 \mathrm{~km} \mathrm{ss}^{-1}$.

\subsection{Scattering effects}

In a previous paper concerning prominences (Gunár et al. 2012), large parts of the solar limb were visible outside the prominence, so that it was possible to observe scattered $\mathrm{H} \alpha$ profiles directly. This is not the case in the present set of data. It can be noted that the far wings of the line, near the continuum, have almost no tornado/prominence contribution.

We correct for scattered light in the following way: we con sider cuts crossing the limb in channels 1 and 9 along lines parallel to the longer edge of the field stop (the $x$ direction in Fig. 3, top panel). These cuts provide intensity curves that approximately correspond to constant wavelengths. These can be used as standard distributions of stray light outside the solar disc for any wavelength, on the condition that they are normalized by disc intensity and measured at a given distance from the limb (for example $20^{\prime \prime}$ ) along the same direction of constant wavelength.

In all MSDP channels, a correction for stray light is obtained by subtracting such normalized intensity functions along cuts parallel to the longer edge of field stop. This correction is a lower limit for the stray light level because the disc radius is larger in the $\mathrm{H} \alpha$ profile than it is in the continuum. It is also a rough approximation because the relative limb darkening is not exactly the same at all wavelengths.

\subsection{Relative intensity and profiles}

After having applied all calibrations and corrections, we did not use the coordinate system of observations $(x, y)$ further. For Fig. 1, where the tornado is presented in a general context, we have co-aligned the MSDP and SDO images and we adopted heliographic coordinates. There is a rotation of -10 degrees with the $(x, y)$ coordinates. To obtain the evolution of the Doppler shifts versus time we computed time distance diagrams and again rotated the field of view of the data cubes to have one axis parallel to the limb. It is this reference system that is used in Figs. 4-7.
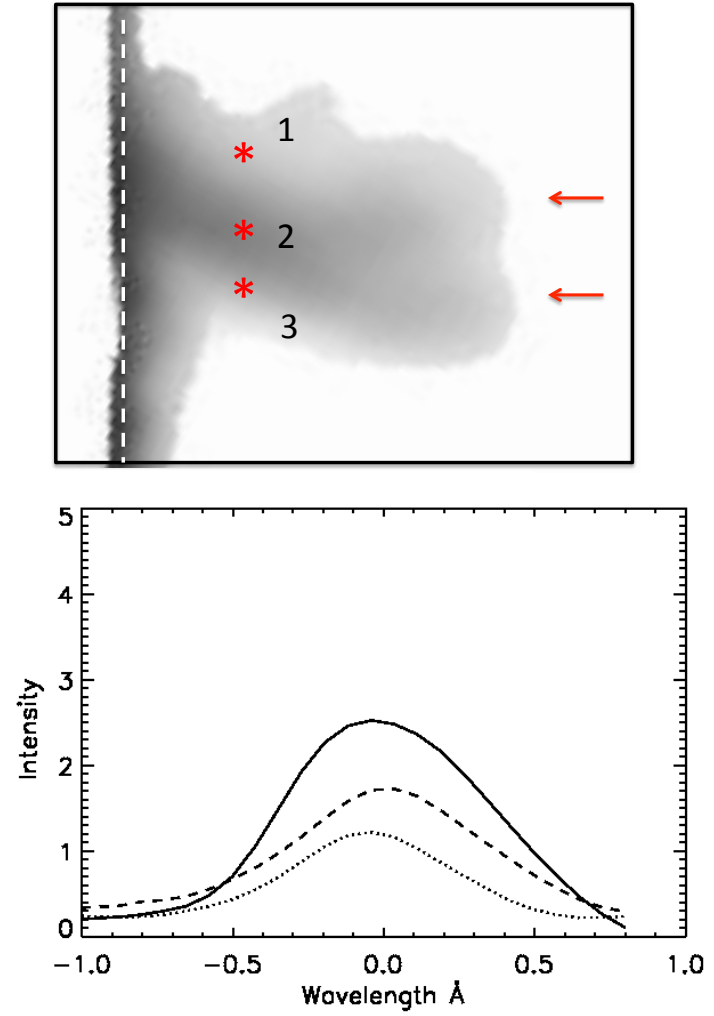

Fig. 4. Top panel: $\mathrm{H} \alpha$ integrated intensity map of the tornado observed with the Meudon MSDP. The field of view is $70^{\prime \prime} \times 45^{\prime \prime}$. The dashed line approximately represents the limb. The two red arrows point out the double structure of the tornado at its top. Asteriks 1-3 are the selected pixels for showing examples of profiles. Bottom panel: $\mathrm{H} \alpha$ profiles: pixel 1 dashed line, pixel 2 solid line, and pixel 3 dotted line. The unit of the intensity is $10^{-6} \mathrm{erg} \mathrm{s}^{-1} \mathrm{~cm}^{-2} \mathrm{sr}^{-1} \mathrm{~Hz}^{-1}$.

Figure 4 (top panel) presents an example of the observations of the tornado with the MSDP, and Fig. 4 (bottom panel) shows some typical profiles of $\mathrm{H} \alpha$ in the centre and edges of the tornado. The tornado shows a double structure at its top, as indicated by red arrows in Fig. 4 (top panel).

\subsection{Doppler shifts}

We analysed the spatial variation of $\mathrm{H} \alpha$ Doppler shifts at $\pm 0.30 \AA$ in the tornado to see whether or not we can detect a signature of rotation in the cool plasma $\left(10^{4} \mathrm{~K}\right)$, as is concluded in some studies (Orozco Suárez et al. 2012; Wedemeyer et al. 2013).

Figures 5 and 6 show the dynamical behaviour of the prominence between 12:06 UT and 12:53 UT and between 13:04 UT and 14:02 UT, respectively. The upper panels of these two figures show snapshots of the Doppler maps at different times, revealing the evolution of the redshift and blueshift patterns in the prominence. The values of the Doppler shifts are coherent in areas (5 to $10^{\prime \prime}$ ) that are much larger than the spatial resolution. Such coherent patterns of similar cell sizes have already been found in legs of prominences (Schmieder et al. 2010). The lower panels show the evolution of these velocities over time for the five cuts indicated in the upper panels. These time slice Doppler shift diagrams are notably different depending on which cut is being considered. Looking at the temporal evolution, it appears that the blue regions become red over quasi-periodic intervals, and vice 
12:06 UT
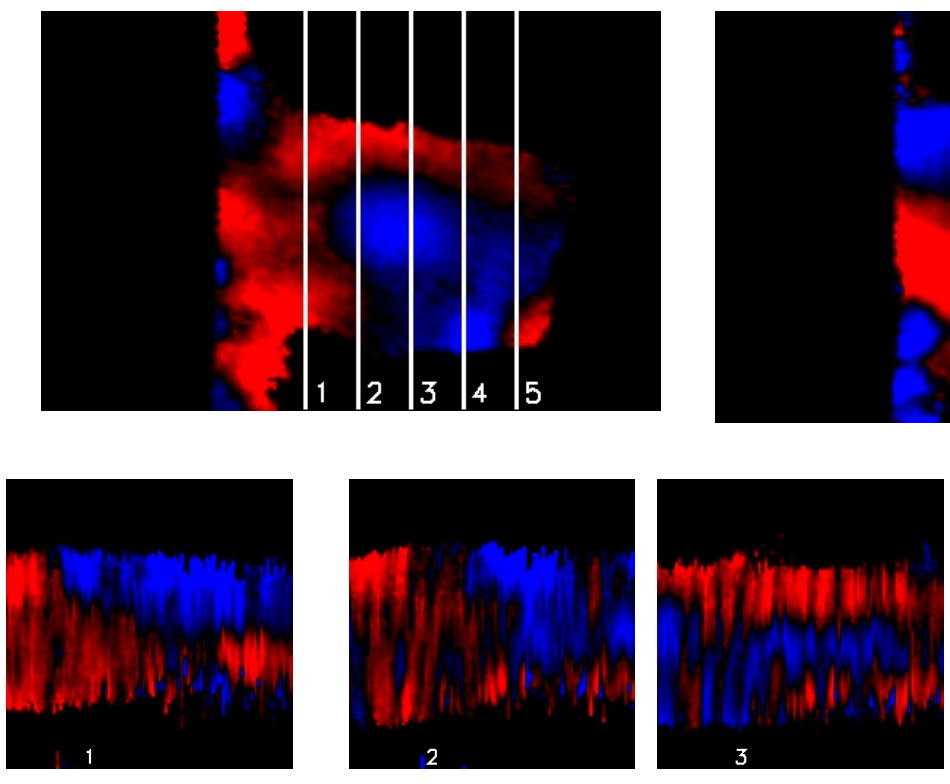

$t$

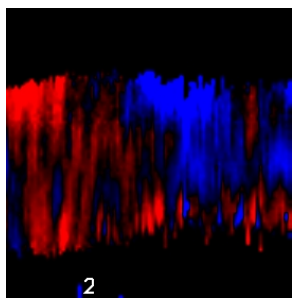

$t$ $t$
$12: 53$ UT
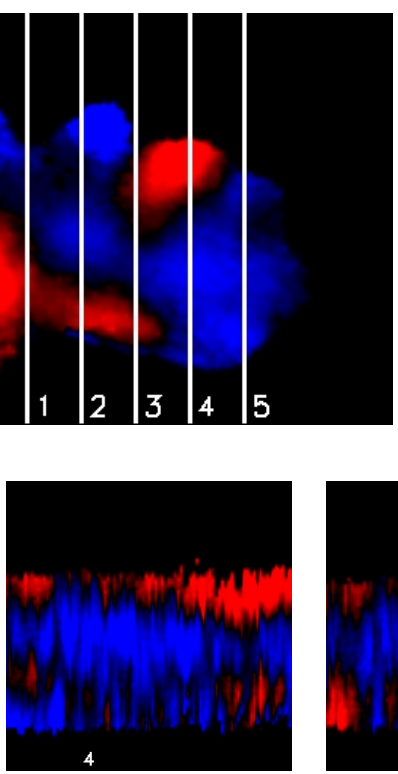

t

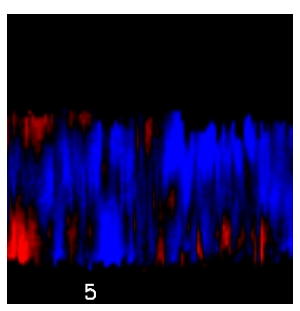

$t$

Fig. 5. Prominence observed on September 24, 2013 by the MSDP operating on the Solar Tower in Meudon. Top panels: Doppler shift maps at 12:06 UT and 12:53 UT and the colour bar (the units are in $\mathrm{km} \mathrm{s}^{-1}$ ). Between blue and red, the black colour corresponds to zero velocity. Blue/red are blue and redshifts, respectively. The limb is vertical on the left of the images. The FOV is $70^{\prime \prime} \times 45^{\prime \prime}$. Bottom panels: time distance Doppler shift diagrams over 58 min for the five cuts ( 1 to 5) shown in the upper panels at a distance of $5^{\prime \prime}$ apart. The time step is $30 \mathrm{~s}$.

13:04 UT

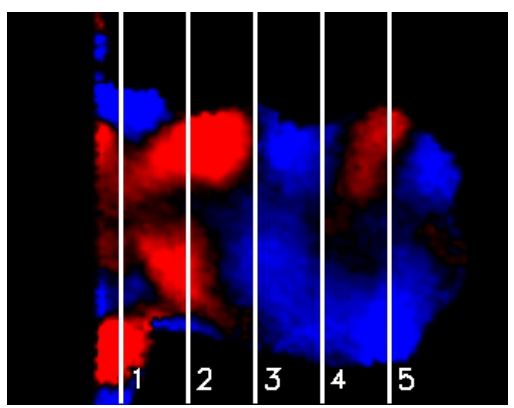

13:35 UT

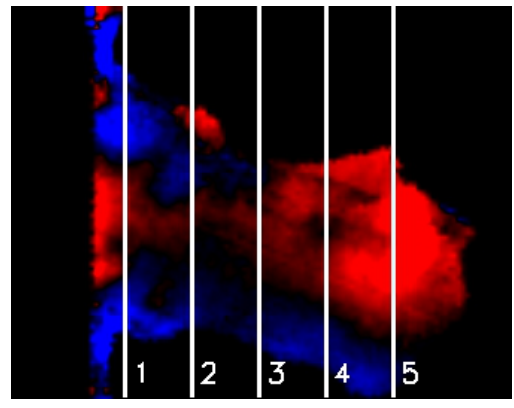

14:02 UT

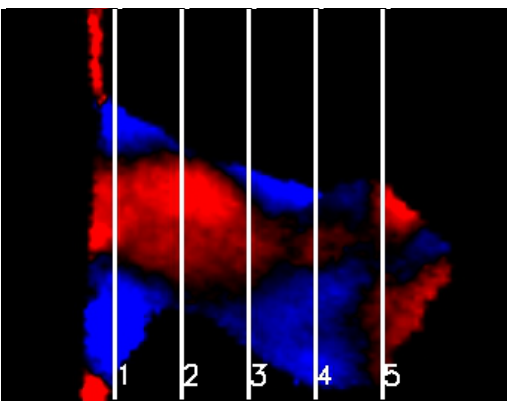

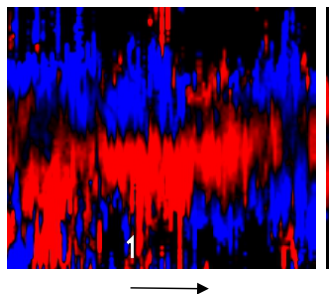

t

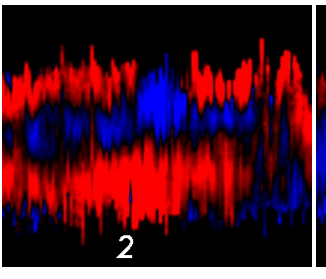

$t$

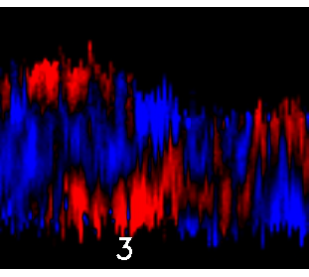

$\mathrm{t}$

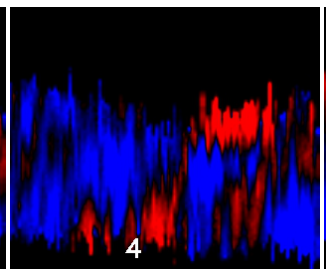

$t$

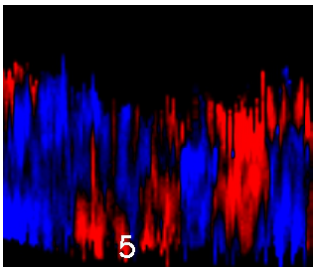

t

Fig. 6. Prominence observed on September 24, 2013 by the MSDP operating on the Solar Tower in Meudon. Top panels: Doppler shift maps at 13:04 UT, 13:35 UT and 14:02 UT. The limb is vertical on the left, and the field of view is 70 " $\times 45^{\prime \prime}$. Bottom panels: time distance diagrams over $47 \mathrm{~min}$ for the five cuts ( 1 to 5 ) shown in the upper panels at a distance of $6^{\prime \prime}$ apart. The time step is $30 \mathrm{~s}$. See the colour bar in Fig. 5 .

versa. After $\sim 20 \mathrm{~min}$ to $30 \mathrm{~min}$ the blue region has again become a red region.

If we consider only one cut during the first part of the sequence, i.e. cut 1 as seen in Fig. 5, we could conclude that we are seeing a rotation of the structure with redshifts on one side of the axis and blueshifts on the other, as found by Orozco Suárez et al. (2012). However, at the end of the sequence and in other cuts, the Doppler shift patterns are difficult to explain in terms of rotation. We can also see that the two parallel structures detected in the intensity map (Fig. 4) behave differently. 

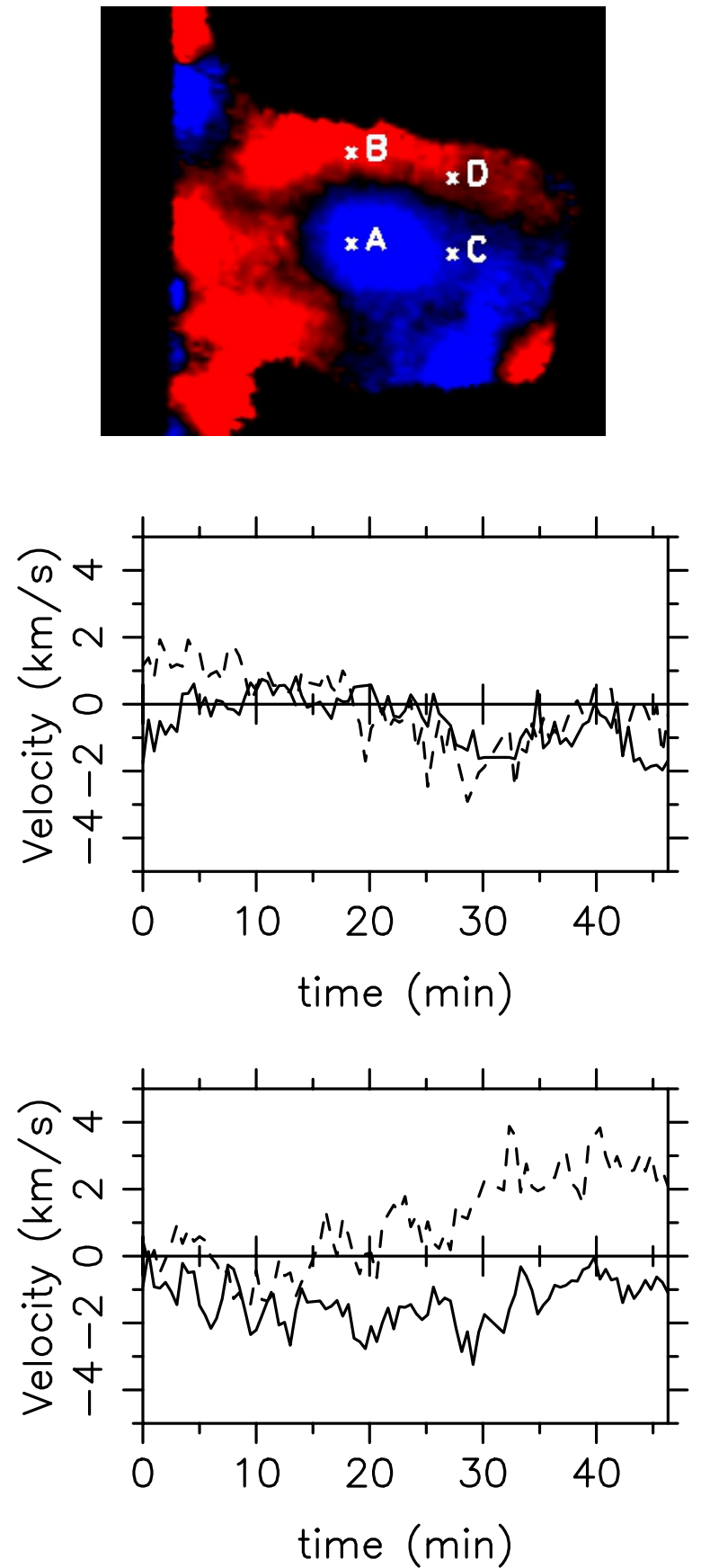

Fig. 7. Evolution of Doppler shifts vs. time between 12:06 UT and 12:53 UT. Top panel: $\mathrm{H} \alpha$ Doppler map, showing four points in the tornadoes (A-D), where the variation of the Doppler shift was measured. Middle panel: variation of the Doppler shift at points A (solid line) and B (dashed line). Bottom panel: variation of the Doppler shift at points $\mathrm{C}$ (solid line) and D (dashed line). See the colour bar in Fig. 5.

Figure 7 shows the velocity behaviour versus time for four selected points (A, B, C, D) in these two structures. Large period oscillations with periods of 40 to $60 \mathrm{~min}$ period are clearly seen. The velocities versus time are not really stochastic. The curves corresponding to points $\mathrm{C}$ and $\mathrm{D}$, in particular, seem to exhibit some periods of the order of 4 or $6 \mathrm{~min}$, which are much larger than the time interval between successive exposures. Evolving solar structures can account for such oscillations.

However the velocity measured at each pixel is a mean value of a number of thread velocities along the line of sight. The $\mathrm{H} \alpha$ line is optically thin and many structures are integrated along

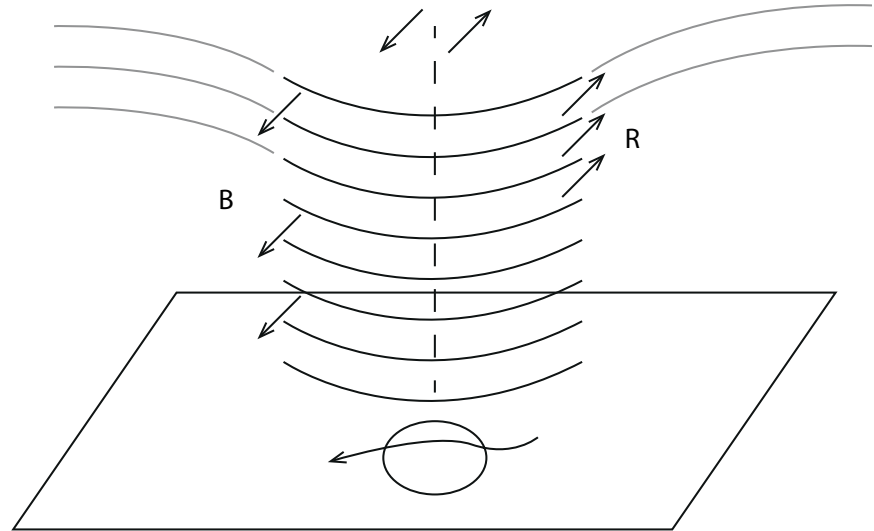

Fig. 8. Sketch of a tornado model for a time during the oscillations. The image shows dipped magnetic field lines of a flux tube attracted by a parasitic polarity (indicated here by the circle in the horizontal plane). "R" means redshift and " $B$ " means blueshift, as seen along that line of sight. The arrows (straight and turning) indicate the direction of motion of the magnetic field lines.

each line of sight, and these structures may have different velocities. Models of multiple threads with random velocity distribution have correctly reproduced $\mathrm{H} \alpha$ profiles (Gunár et al. 2010, 2012; Labrosse \& Rodger 2016).

\subsection{Electron and ion densities}

The top panel of Fig. 4 shows the $\mathrm{H} \alpha$ image of the tornado at 12:22 UT, and corresponds to the prominence shown in the box in Fig. 1. At the top of the tornado we can distinguish two structures, shown in Fig. 4 with two red arrows, which correspond to the two structures visible in AIA that appear to oscillate (Fig., 2, top cut).

Three characteristic $\mathrm{H} \alpha$ profiles are shown in Fig. 4 (bottom panel). The units of intensity are erg s $\mathrm{s}^{-1} \mathrm{~cm}^{-2} \mathrm{sr}^{-1} \mathrm{~Hz}^{-1}$. These profiles have been calibrated using the standard reference profiles defined by David (1961). At disc centre the central intensity of the $\mathrm{H} \alpha$ profile is $I_{\mathrm{c}}=4.077 \times 10^{-5} \mathrm{erg} \mathrm{s}^{-1} \mathrm{~cm}^{-2} \mathrm{sr}^{-1} \mathrm{~Hz}^{-1}$. The ratio of the peak intensity of the centre of the prominence, $I_{\text {peak }}$, to disc centre intensity, $I_{\mathrm{c}}$, is $\mathrm{I}_{\text {peak }} / I_{\mathrm{c}}=0.25 / 4.077=6.1 \%$

According to the graph in Wiik et al. (1992, their Fig. 10) this corresponds to an electron density, $n_{\mathrm{e}}$, in the range $2-4 \times$ $10^{10} \mathrm{~cm}^{-3}$. The height at which these values are measured (black arrow, Fig. 4, lower panel) is at a much lower altitude than the $45 \mathrm{Mm}$ that was used in the calculations of Wiik et al. (1992), so these values may be reduced slightly.

The integrated intensity $\left(I_{\text {int }}=\sum I_{\nu} \mathrm{d} v=\sum I_{\nu} c / \lambda^{2} \mathrm{~d} \lambda\right)$ of the tornado from $\mathrm{H} \alpha$ profiles were computed for the following three locations in the tornado:

south edge: $1.1 \times 10^{5} \mathrm{erg} \mathrm{s}^{-1} \mathrm{~cm}^{-2} \mathrm{sr}^{-1}$;

centre: $1.5 \times 10^{5} \mathrm{erg} \mathrm{s}^{-1} \mathrm{~cm}^{-2} \mathrm{sr}^{-1}$;

north edge: $0.74 \times 10^{5} \mathrm{erg} \mathrm{s}^{-1} \mathrm{~cm}^{-2} \mathrm{sr}^{-1}$.

These values were compared to the model of Gouttebroze et al. (1993) to see what total hydrogen density, $n_{\mathrm{H}}$, it gives. The values are close to some of the models and seem to suggest that we have a total hydrogen density of $n_{\mathrm{H}}=1.8 \times 10^{11} \mathrm{~cm}^{-3}$ for $T=6000 \mathrm{~K}, p=0.2 \mathrm{dyn}^{-2}$ and $D=1000 \mathrm{~km}$, which is similar to the values for $T, D$ and $p$ that were estimated in Levens et al. (2016a). The peak intensity from the model is $3.01 \times 10^{-6} \mathrm{erg} \mathrm{s}^{-1} \mathrm{~cm}^{-2} \mathrm{sr}^{-1} \mathrm{~Hz}^{-1}$ (integrated intensity, $I_{\text {int }}=1.14 \times 10^{5} \mathrm{erg} \mathrm{s}^{-1} \mathrm{~cm}^{-2} \mathrm{sr}^{-1}$ ), where we find a value of 
$2.5 \times 10^{-6} \mathrm{erg} \mathrm{s}^{-1} \mathrm{~cm}^{-2} \mathrm{sr}^{-1} \mathrm{~Hz}^{-1}$ at the centre of the tornado. This is consistent with previously measured values. It leads to a plasma- $\beta$ of $3 \times 10^{-3}$, confirming that the magnetic pressure dominates over the gas pressure. The total hydrogen density is an important parameter for the estimation of the Alfvén speed in the prominence plasma in the context of waves that could be used to explain the oscillations.

\section{Discussion and conclusions}

A tornado-like structure was observed on September 24, 2013 by AIA with a rotating period of around 90 minutes as it crossed the limb. At the Meudon Solar Tower this tornado prominence was observed for a few hours with the MSDP spectrograph in $\mathrm{H} \alpha$ with a high cadence (two images per minute). Because of its concept of a large open slit at the entrance of the spectrograph, the MSDP allows us to have 3D data cubes of intensity in two coordinates and $\lambda$, and to recover Doppler shift data cubes in two coordinates and time by processing a long sequence of observations.

We analysed the spatial variation of the Doppler shifts in the prominence to see if we can detect rotational motion in the cool plasma. The Doppler shift maps present a pattern of small areas of alternating blueshifts and redshifts, which evolve over time. We can detect a double structure parallel to its central axis. Looking at the temporal evolution, it appears that the blue region becomes red with a quasi-periodicity of 40 to $60 \mathrm{~min}$. Superimposed onto these oscillations are small amplitude oscillations with periods between 4 and 6 min.

The behaviour of the $\mathrm{H} \alpha$ Doppler shift pattern is very different from the EIS observations, which show persistent blueshifts and redshifts symmetrically about the tornado axis ( $\mathrm{Su}$ et al. 2014; Levens et al. 2015). The capabilities of the MSDP instrument offer the possibility of looking at the temporal evolution of the Doppler shifts at any position in the FOV. With EIS, the few studies that seem to point to rotation are much more limited in the sense that the sit-and-stare is only at one location. This would be equivalent to taking, for example the time-slice diagram at cut 1 in Fig. 5 or cut 3 in Fig. 6 and saying that this is proof of rotation. Now, with the MSDP we show that, by looking at several locations, the data is not consistent with rotation. This is, so far, a unique analysis of Doppler shifts in a 3D data cube $(x, y, t)$ with a high cadence (two images per minutes).

The $\mathrm{H} \alpha$ velocity behaviour gives some constraints on the models proposed to interpret the rotation of AIA tornadoes. A first scenario could be to interpret the quasi-periodic pattern observed by AIA as being due to kink oscillations such as in Mghebrishvili et al. (2015). Such a scenario leads those authors to derive some physical characteristics of their tornado. In particular they found a magnetic field around $B=5$ gauss (Mghebrishvili et al. 2015).

However we already have some indications about the magnetic field measured in a tornado by THEMIS (Schmieder et al. 2015; Levens et al. 2016b). The magnetic field strength has been found to be between 10 and 45 gauss and is not as weak as in quiescent prominences ( $B=5$ gauss). Considering the results that we get in the present analysis of the tornado of September 24, 2013 we can estimate the phase speed of the apparent motions. The quasi-periodicity of the oscillations perpendicular to its vertical axis seen with AIA is around 90 min. Preliminary calculations of transverse oscillations for cool plasma would lead to the following estimation: the sound speed is $\sim 7 \mathrm{~km} \mathrm{~s}^{-1}$ (based on $T=6000 \mathrm{~K}$ for the prominence plasma). The Alfvén speed is $\sim 127 \mathrm{~km} \mathrm{~s}^{-1}$ (using a mean value $B=25 \mathrm{G}$, pressure $p=0.3$ dyn $\mathrm{cm}^{-2}$, and $T=6000 \mathrm{~K}$, giving a total hydrogen density of $n_{\mathrm{H}}=1.8 \times 10^{11} \mathrm{~cm}^{-3}$ ). If this is a kink oscillation, then the phase speed should be higher by $\sim \sqrt{2}$, assuming that the density outside the prominence is very small compared to the prominence material density, and we get the kink speed, $c_{k}=179 \mathrm{~km} \mathrm{~s}^{-1}$.

The phase speed of the wave from the fundamental mode can be estimated (from e.g. coronal seismology, see review by Nakariakov \& Verwichte 2005) as $c=2 L / P=2 \times 10^{5} /(90 \times$ $60)=37 \mathrm{~km} \mathrm{~s}^{-1}$, where $P$ is the oscillation period and $L$ is the length of the oscillating thread. If the thread is not oscillating at the fundamental mode, then the wavelength could be shorter by the mode number factor. For example, assuming a full wavelength, we get $c=18.5 \mathrm{~km} \mathrm{~s}^{-1}$. The estimated phase speed from the apparent oscillation is too high to match either the sound nor Alfvén speed according to the values of temperature, density of the cool $\mathrm{H} \alpha$ plasma that we determined spectroscopically, and the estimated magnetic field strength of tornadoes. This solution could be valid only for extreme values of $B$ or $L$. The $L$ value would be the full length of the prominence plus the tornado (see Fig. 1) and $B$ should be lowered by a factor 2 to 4 . We do not believe that $B$ in tornadoes can be reduced to 5 gauss because of our $B$ measurements in tornadoes presented in previous papers (Schmieder et al. 2015; Levens et al. 2016b). The conclusion of this analysis is that the pure kink mode wave interpretation is difficult to be justified in our case.

Another solution would be to consider that the oscillations are from plasma motions along the magnetic field. The orientation of the magnetic field in tornadoes was found mainly horizontal (Schmieder et al. 2015; Levens et al. 2016b). If we consider longitudinal waves along the field lines, as in Terradas et al. (2015) and Luna et al. (2016), the Doppler shifts would indicate a component of the velocity along the line of sight, which is more or less perpendicular to the direction of the horizontal field, if we assume that the tornado is connected to the northern feet of the prominence by horizontal field lines, parallel to the solar limb. This would mean that the velocity of the plasma along the field is much larger than the $2 \mathrm{~km} \mathrm{~s}^{-1}$ that has been measured. The pendulum model, where plasma is moving along rigid magnetic field lines, could explain the oscillations (Luna et al. 2016). This orientation could account for both the plane-of-sky motion seen in AIA and the oscillation signatures seen in the Doppler maps from MSDP. The period of the oscillation would depend on the curvature of the dipped field lines that support the plasma. The different periods registered along the prominence could be due to the different curvatures. In that case the oscillations observed by AIA and differences in the direction of the Doppler velocities in $\mathrm{H} \alpha$ across the tornado would be caused by counter-streaming, as was discussed in Panasenco et al. (2014). It is, however, difficult to justify that the field lines are rigid and do not move in such a dynamic atmosphere that is governed by the magnetic pressure.

We now discuss MHD sketch models. The transverse displacement shown in AIA should also be along the line of sight, and it should deform the magnetic field slightly. The azimuth would be an interesting parameter to study over time. However we have no evolution of the magnetic field parameters over time during the present observation because of the low temporal resolution of THEMIS; it takes one hour to measure the Stokes parameters for a field of view of $120^{\prime \prime} \times 160^{\prime \prime}$. To combine the observations of the transverse oscillations seen in AIA and the oscillations of the $\mathrm{H} \alpha$ plasma, we need to consider horizontal magnetic field lines that have an angle with the line of sight, somewhere around $45^{\circ}$. The dip field lines would therefore not be in the plane of the sky, but viewed from an angle. 
Tornadoes are probably the legs of prominences, as has been suggested previously (Wedemeyer et al. 2013; Levens et al. 2016a), and could be modelled as a pile up of dips supporting the cool plasma in the corona. The legs (barbs, footpoints) are directly related to parasitic polarities (Aulanier \& Demoulin 1998). Schmieder et al. (2014a) demonstrate that the polarities related to the anchorage of the prominence legs are at the borders of the supergranules and generally at the convergence point between two supergranules. The diffusion of polarities appearing in the internetwork or cancelling flux are permanent motions in the dynamic photosphere. The piles of dips would move from left to right with some kind of oscillatory motions (Fig. 8).

The main conclusion of this paper is that the H $\alpha$ Doppler shift pattern of the tornado observed simultaneously by AIA in $193 \AA$ cannot be interpreted in terms of rotation. We therefore look forward to new observing campaigns involving ground-based instruments and Hinode (SOT and EIS) and IRIS to measure the Doppler shifts over a large temperature range to better understand the dynamical coupling between different plasmas in and around the prominence. The MHD simulations of oscillations in a realistic, three-dimensional magnetic field, and density model of a prominence would be suitable to disentangle the different mechanisms that have been suggested by these observations.

Acknowledgements. The authors would like to thank the anonymous referee for his/her fruitful comments, R. Lecocguen, D. Crussaire and the team at the MSDP for acquiring the $\mathrm{H} \alpha$ observations. We thank G. Aulanier for fruitful discussions and the sketch of a "tornado", which has been drawn by Sylvain Cnudde. We deeply thank L. Fletcher for giving many comments on the manuscript to improve its clarity. P.J.L. acknowledges support from an STFC Research Studentship ST/K502005/1. N.L. acknowledges support from STFC grant ST/L000741/1. L.O. would like to acknowledge support by NASA Cooperative Agreement grant NNG11PL10A to CUA. The AIA data are provided courtesy of NASA/SDO and the AIA science team.

\section{References}

Antolin, P., Okamoto, T. J., De Pontieu, B., et al. 2015, ApJ, 809, 72

Anzer, U., \& Heinzel, P. 2005, ApJ, 622, 714

Aulanier, G., \& Demoulin, P. 1998, A\&A, 329, 1125

Berger, T. 2014, in IAU Symp. 300, eds. B. Schmieder, J.-M. Malherbe, \& S. T. Wu, 15

Chae, J. 2010, ApJ, 714, 618

David, K.-H. 1961, Z. Astrophys., 53, 37

Dudík, J., Aulanier, G., Schmieder, B., Bommier, V., \& Roudier, T. 2008, Sol. Phys., 248, 29
Dudík, J., Aulanier, G., Schmieder, B., Zapiór, M., \& Heinzel, P. 2012, ApJ, 761,

Engvold, O. 2008, in Waves \& Oscillations in the Solar Atmosphere: Heating and Magneto-Seismology, eds. R. Erdélyi, \& C. A. Mendoza-Briceno, IAU Symp., 247, 152

Gouttebroze, P., Heinzel, P., \& Vial, J. C. 1993, A\&AS, 99, 513

Gunár, S., Schwartz, P., Schmieder, B., Heinzel, P., \& Anzer, U. 2010, A\&A, 514, A43

Gunár, S., Mein, P., Schmieder, B., Heinzel, P., \& Mein, N. 2012, A\&A, 543, A93

Heinzel, P., \& Anzer, U. 1999, Sol. Phys., 184, 103

Heinzel, P., Schmieder, B., Mein, N., \& Gunár, S. 2015, ApJ, 800, L13

Kosugi, T., Matsuzaki, K., Sakao, T., et al. 2007, Sol. Phys., 243, 3

Labrosse, N., \& Rodger, A. S. 2016, A\&A, 587, A113

Lemen, J. R., Title, A. M., Akin, D. J., et al. 2012, Sol. Phys., 275, 17

Levens, P. J., Labrosse, N., Fletcher, L., \& Schmieder, B. 2015, A\&A, 582, A27

Levens, P. J., Schmieder, B., Labrosse, N., \& López Ariste, A. 2016a, ApJ, 818, 31

Levens, P. J., Schmieder, B., López Ariste, A., et al. 2016b, ApJ, 826, 164

López Ariste, A. 2015, in IAU Symp. 305, eds. K. N. Nagendra, S. Bagnulo, R. Centeno, \& M. Jesús Martínez González, 207

Luna, M., Moreno-Insertis, F., \& Priest, E. 2015, ApJ, 808, L23

Luna, M., Terradas, J., Khomenko, E., Collados, M., \& de Vicente, A. 2016, ApJ, 817,157

Mackay, D. H., Karpen, J. T., Ballester, J. L., Schmieder, B., \& Aulanier, G. 2010, Space Sci. Rev., 151, 333

Mein, P. 1991, A\&A, 248, 669

Mghebrishvili, I., Zaqarashvili, T. V., Kukhianidze, V., et al. 2015, ApJ, 810, 89

Nakariakov, V. M., \& Verwichte, E. 2005, Living Rev. Sol. Phys., 2, 3

Ofman, L., Kucera, T. A., Mouradian, Z., \& Poland, A. I. 1998, Sol. Phys., 183, 97

Ofman, L., Knizhnik, K., Kucera, T., \& Schmieder, B. 2015, ApJ, 813, 124

Okamoto, T. J., Tsuneta, S., Berger, T. E., et al. 2007, Science, 318, 1577

Okamoto, T. J., Antolin, P., De Pontieu, B., et al. 2015, ApJ, 809, 71

Orozco Suárez, D., Asensio Ramos, A., \& Trujillo Bueno, J. 2012, ApJ, 761, L25

Panasenco, O., Martin, S. F., \& Velli, M. 2014, Sol. Phys., 289, 603

Schmieder, B., Lin, Y., Heinzel, P., \& Schwartz, P. 2004, Sol. Phys., 221, 297

Schmieder, B., Chandra, R., Berlicki, A., \& Mein, P. 2010, A\&A, 514, A68

Schmieder, B., Kucera, T. A., Knizhnik, K., et al. 2013, ApJ, 777, 108

Schmieder, B., Roudier, T., Mein, N., et al. 2014a, A\&A, 564, A104

Schmieder, B., Tian, H., Kucera, T., et al. 2014b, A\&A, 569, A85

Schmieder, B., López Ariste, A., Levens, P., Labrosse, N., \& Dalmasse, K. 2015, in IAU Symp. 305, eds. K. N. Nagendra, S. Bagnulo, R. Centeno, \& M. Jesús Martínez González, 275

Su, Y., Gömöry, P., Veronig, A., et al. 2014, ApJ, 785, L2

Terradas, J., Soler, R., Luna, M., Oliver, R., \& Ballester, J. L. 2015, ApJ, 799, 94

Wedemeyer, S., Scullion, E., Rouppe van der Voort, L., Bosnjak, A., \& Antolin, P. 2013, ApJ, 774, 123

Wiik, J. E., Heinzel, P., \& Schmieder, B. 1992, A\&A, 260, 419

Xia, C., Keppens, R., Antolin, P., \& Porth, O. 2014, ApJ, 792, L38 